\title{
ON SOME SHRINKAGE TECHNIQUES FOR ESTIMATING THE PARAMETERS OF EXPONENTIAL DISTRIBUTION
}

\author{
M. A. Q. YOUSEF, M. S. ABU-SALIH and M. A. ALI \\ Yarmouk University, Irbid, Jordan \\ Key words : Shrinkage techniques, exponential distribution
}

\begin{abstract}
A variety of shrinkage methods have been proposed for estimation of some unknown parameter by considering estimators based on a prior guess of the value of the parameter. We compare some of the shrunken estimators for the parameters $\mu$ and $\theta$ of the exponential distribution through simulation.
\end{abstract}

\section{INTRODUCTION}

In the estimation of an unknown parameter there often exists some form of prior knowledge about the parameter which one would like to utilize in order to get a better estimate. Thompson ( 1968 ) described a shrinkage technique for estimating the mean of a population. Mehta and Srinivasan ( 1971 ) proposed another class of shrunken estimator for the mean of a population and have shown that this class has better performance than that of Thompson ( 1968 ) in terms of mean squared error. Pandey and Singh ( 1977 ) and Pandey ( 1979 ) described shrinkage techniques for estimating the variance of a normal population. Lemmer (1981) considered a shrunken estimator for the parameter of the binomial distribution. His estimator is similar to the Pandey ( 1979 ) estimator for the variance of a normal distribution.

We consider a variety of shrinkage methods for estimating the parameters $\mathrm{u}$ and $\mathbf{0}$ of the exponential distribution. These estimators are compared through simulation.

\section{ESTIMATORS CONSIDERED}

Let the length of life $\mathrm{X}$ of a certain system be distributed as

$$
f(x, \theta, \mu)=\frac{1}{\theta} \exp [-(x-\mu) / \theta], \quad 0 \leqslant \mu \leqslant x, \quad \theta>0
$$


A random sample of $\mathbf{n}$ such systems is subjected to test and the test terminated as soon as the first $r(\leqslant n)$ items fail. Let $\underset{\sim}{x}=\left\{x_{(1)}<\ldots,<x_{(r)}\right\}$ be the first $r$ ordered failure times. It is well known from Epstein and Sobel ( 1954$)$ that

and

$$
\hat{\theta}=\left[\sum_{i=1}^{r} x_{(i)}+(n-r) x_{(r)}-n x_{(1)}\right] /(r-1), r>1
$$

$$
\hat{\mu}=x_{(1)}-\hat{\theta} / n,
$$

are the minimum variance unbiased estimators of $\theta$ and $u$ respectively. The variances of these estimators age given by

$$
\begin{gathered}
\operatorname{var}(\hat{\theta})=\theta^{2} /(r-1) \\
\operatorname{var}(\hat{\mu})=r \theta^{2} / n^{2}(r-1)
\end{gathered}
$$

( see Bain ( 1978 ), p - 163 ).

The first estimator considered is :

$$
\stackrel{v}{\mu}_{T}=\mu_{0}+C\left(\hat{\mu}-\mu_{0}\right) \quad 0 \leqslant c \leqslant 1
$$

where $\mu_{0}$ is the guessed value of $\mu_{.}^{v} \mu_{T}$ is the actual Thompson-type estimator. Thompson suggested to determine $\mathrm{C}$ from

$$
\frac{\partial \operatorname{MSE}\left(\mathrm{u}_{\mathrm{T}}^{\mathrm{v}}\right)}{\partial_{\mathrm{C}}}=0,
$$

with $\operatorname{MSE}\left(\stackrel{v}{\mu_{T}}\right)=E\left(\mu_{T}-\mu\right)^{2}$, the mean squared error of $\stackrel{v}{\mu}_{T}$. It follows that

$$
C=\left(\mu-\mu_{0}\right)^{2} /\left[\left(\mu-\mu_{0}\right)^{2}+\operatorname{var}(\hat{\mu})\right]
$$

In practice $C$ in (2.2) is estimated by replacing the unknown parameters by their sample estimates. Substituting the estimated value of $C$ in (2.1) we have

$$
\hat{\mu}_{T}=\mu_{o}+(\hat{\mu}-\mu)^{3} /\left[\left(\hat{\mu}-\mu_{0}\right)^{2}+r \hat{\theta}^{2} / n^{2}(r-1)\right]
$$

Secondly, we consider the Mehta and Srinivasan-type estimator (cf. Mehta and Srinivasan ( 1971 ) for $\mu$ : 


$$
\hat{\mu}_{M}=\hat{\mu}-a\left(\hat{\mu}-\mu_{0}\right) \exp \left[-b n^{2}(r-1)\left(\hat{\mu}-\mu_{o}\right) / r \hat{\theta}^{2}\right],
$$

where $\mathrm{a}$ and $\mathrm{b}$ are suitably chosen positive constants $\mathrm{a}<\mathrm{b}$. No general guidance has been given on how $a$ and $b$ should be chosen.

Now we consider the Pandey-type estimator of $\mu$ :

$$
\stackrel{v}{\mu}_{P}=a\left[K \mu+(1-K) \mu_{o}\right], \quad 0 \leqslant K \leqslant 1
$$

with $\mathrm{K}$ a constant specified by the experimentor according to his belief in $\mu_{\mathrm{o}}$ and $\mathrm{a}$ is determined from $\partial \mathrm{MSE}\left(\mathrm{v}_{\mathrm{p}}\right) / \partial \mathrm{a}=0$. It follows that $a=d_{1} \mu^{2}$ $/\left[K^{2} \operatorname{var}(\hat{\mu})+d_{1} \mu^{2}\right]$ where $d_{1}=K+(1-K) \mu_{0} / \mu$. Usually a is estimated by replacing the unknown parameters by their sample estimates.

Substituting the estimated value of a in ( 2.5 ) we obtain

$$
\hat{\mu}_{P}=\hat{d}_{1}^{2} \hat{\mu}^{3} /\left[\hat{d}_{1}^{2} \hat{\mu}^{2}+K^{2} r \hat{\theta}^{2} / n^{2}(r-1)\right]
$$

with

$$
\hat{\mathrm{d}}_{1}=\left[\mathrm{K}+(1-\mathrm{K}) \mu_{\mathrm{o}} / \hat{\mu}\right]
$$

Finally, we consider Lemmer-type estimator ( cf. Lemmer ( 1981 )) for $\mu$ :

$$
\hat{\mu}_{L}=K \hat{\mu}+(1-K) \mu_{0}
$$

which follows from (2.5) if $a=1$. Of all estimators considered, $\hat{\mu}_{L}$ is the simplest. As $\hat{\mu}_{P}$ and $\hat{\mu}_{L}$ depend on $K$, different values of $K$ have been considered.

All the above approaches can be used to define variety of shrunken estimators for the parameter $\theta$. We present all the estimators considered in the following table.

\section{COMPARISON OF ESTIMATORS}

Simulation experiments are used to estimate the mean squared errors for the five estimators of $\mu$ and $\theta$. The procedure is described below : 
Table 1

Shrunken Estimators For $\mathbf{u}$ and $\boldsymbol{\theta}$

\begin{tabular}{|c|c|c|}
\hline Parameter & $\begin{array}{c}\text { Type of } \\
\text { Estimator }\end{array}$ & Estimator \\
\hline $\begin{array}{l}\text { Location } \\
\text { Parameter } \\
\mu\end{array}$ & $\begin{array}{l}\text { Thompson } \\
\text { Mehta-Srinivasan } \\
\text { Pandey } \\
\text { Lemmer }\end{array}$ & $\begin{array}{l}\hat{\mu}_{T}=\mu_{o}+\left(\hat{\mu}-\mu_{o}\right)^{3} /\left[\left(\hat{\mu}-\mu_{o}\right)^{2}+r \hat{\theta}^{2} /\left\{n^{2}(r-1)\right\}\right] \\
\hat{\mu}_{M}=\hat{\mu}-a\left(\hat{\mu}-\mu_{o}\right) \exp \left[-\left\{b n^{2}(r-1)\left(\hat{\mu}-\mu_{o}\right) / r \hat{\theta}^{2}\right\}\right] \\
\hat{\mu}_{P}=\hat{d}_{1}^{2} \hat{\mu}^{3} /\left[\hat{d}^{2} \hat{\mu}^{2}+K^{2} r \hat{\theta}^{2} / n^{2}(r-1)\right] \\
\hat{\mu}_{L}=K \hat{\mu}+(1-K) \mu_{o}\end{array}$ \\
\hline $\begin{array}{l}\text { Scale } \\
\text { parameter } \\
\theta\end{array}$ & $\begin{array}{l}\text { Thompson } \\
\text { Mehta-Sirivasan } \\
\text { Pandey } \\
\text { Lemmer }\end{array}$ & $\begin{array}{l}\hat{\theta}_{T}=\theta_{0}+\left(\hat{\theta}-\theta_{0}\right)^{3} /\left[\left(\hat{\theta}-\theta_{0}\right)^{2}+\hat{\theta}^{2} /(\mathrm{r}-1)\right] \\
\hat{\theta}_{M}=\hat{\theta}-\mathrm{a}\left(\hat{\theta}-\theta_{0}\right) \exp \left[-\left\{\mathrm{b}(\mathrm{r}-1)\left(\hat{\theta}-\theta_{0}\right) / \hat{\theta}^{2}\right\}\right] \\
\hat{\theta}_{P}=\hat{\mathrm{d}}_{2}^{2} \mu^{3} /\left[\hat{\mathrm{d}}_{2}^{2} \hat{\theta}^{2}+\mathrm{K}^{2} \hat{\theta}^{2} /(\mathrm{r}-1)\right] \\
\hat{\theta}_{\mathrm{L}}=\mathrm{K} \hat{\theta}+(1-\mathrm{K}) \theta_{0}\end{array}$ \\
\hline
\end{tabular}

$\hat{\mathrm{d}}_{1}=\mathrm{K}+(1-\mathrm{K}) \mu_{0} / \hat{\mu}, \quad \hat{\mathrm{d}}_{2}=\mathrm{K}+(1-\mathrm{K}) \theta_{0} / \hat{\theta}, \mathrm{K}$ is a known constant between zero and one, a and $\mathrm{b}$ are positive constants $a<b, \mu_{o}$ and $\theta_{o}$ are the guessed values for $\mu$ and $\theta$ respectively. 
We generate a random sample of size $\mathrm{n}$ from a two-patameter exponential distribution,

$$
f(x, \theta, \mu)=\frac{1}{\theta} \exp [-(x-\mu) / \theta], \quad 0 \leqslant \mu \leqslant x, \quad \theta>0
$$

with $\mu=80$, and $\theta=7.0$. The vector

$$
\mathrm{x}=\left\{\mathrm{x}_{(1)}<\mathrm{x}_{(2)}<\ldots<\mathrm{x}_{(\mathrm{r})}\right\}
$$

of the first $r$-ordered observation is recorded. Then the minimum variance unbiased estimators $\hat{\mu}$ and $\hat{\theta}$ of $\mu$ and $\theta$ respectively are computed using the following formulas.

$$
\hat{\mu}=x_{(1)}-\hat{\theta} / n
$$

and

$$
\hat{\theta}=\left[\sum_{i=1}^{r} x_{(i)}+(n-r) x_{(r)}-n x_{(1)}\right] /(r-1) .
$$

For a known constant $K$ between zero and one and for specific values of $\mu_{0}$ and $\theta_{0}$, the quantities

$$
\hat{\mathrm{d}}_{1}=\mathrm{K}+(1-\mathrm{k}) \mu_{\mathrm{o}} / \hat{\mu} \text { and } \hat{\mathrm{d}}_{2}=\mathrm{K}+(1-\mathrm{K}) \theta_{\mathrm{o}} / \hat{\theta}
$$

are obtained. Then the estimators $\hat{\mu}_{T}, \hat{\mu}_{M}, \hat{\mu}_{P}$ and $\hat{\mu}_{L}$ of $u$ are computed using the relations (2.3), (2.4), (2.6) and (2.7). Similarly, the estimators $\hat{\theta}_{\mathrm{T}}, \hat{\theta}_{\mathrm{M}}, \hat{\theta}_{\mathrm{P}}$ and $\hat{\theta}_{L}$ of $\theta$ are obtained using the formulas shown in Table -1 .

Monte Carlo experiments are repeated 500 times. The average of the 500 sample values of each squared error, e.g. $(\hat{\mu}-\mu)^{2}$, is taken as an estimate of the corresponding mean squared error which is denoted by $\operatorname{MSE}($. ).

The estimates of the mean squared errors of the various estimators of $\mu$ and $\theta$ and the relative efficiencies, e.g.

$$
\operatorname{R}\left(\hat{\mu}_{T} / \hat{\mu}\right)=\operatorname{MSE}\left(\hat{\mu}_{T}\right) / \operatorname{MSE}(\hat{\mu})
$$


are calculated for $\mathrm{n}=30, \mathrm{r}=10,20,30, \mathrm{~K}=0.20,0.70, \mathrm{a}=1,5, \mathrm{~b}=20,50$, $\mu=80, \theta=7.0, \mu_{0}=70$, and $\theta_{0}=5.0$.

Results of the simulation experiments are given in Table $2-3$.

\section{CONCLUSION}

Although the results derived above apply strictly to only very limited cases, they are suggestive of some general conclusions regarding the relative efficiencies of the various methods. Note from Tables 2 that the MSE of $\hat{\mu}_{T}$ are always smaller than that of other estimators. It is obvious that $\hat{\mu}_{T}, \hat{\mu}_{M}$, and $\hat{\mu}_{L}$ have smaller mean squared error than the minimum variance unbiased estimator $\hat{\mu}$.

The mean squared error of $\hat{\mu}_{P}$ is always higher than the MSE of $\hat{\mu}$. The advantages of $\hat{\mu}_{T}$ and $\hat{\mu}_{L}$ are most marked when $r$ is small.

Further, the comparison statistics in Table -3 show that the MSE of $\hat{\theta}_{\mathrm{T}}, \hat{\theta}_{\mathrm{M}}$, and $\hat{\theta}_{L}$ are always smaller than the MSE of $\hat{\theta}$, the minimum variance unbiased estimator. The mean squared error of $\hat{\theta}_{P}$ is always greater than the MSE of $\hat{\theta}$. The MSE of Thompson-type estimator is smaller than those of the remaining estimators. 
Table 2

Relative Efficiencies of Various Shrunken Estimators of $\mu$

Sample size $n=30, \mu=80, \theta=7.0, \mu_{0}=70, \theta_{0}=5.0$

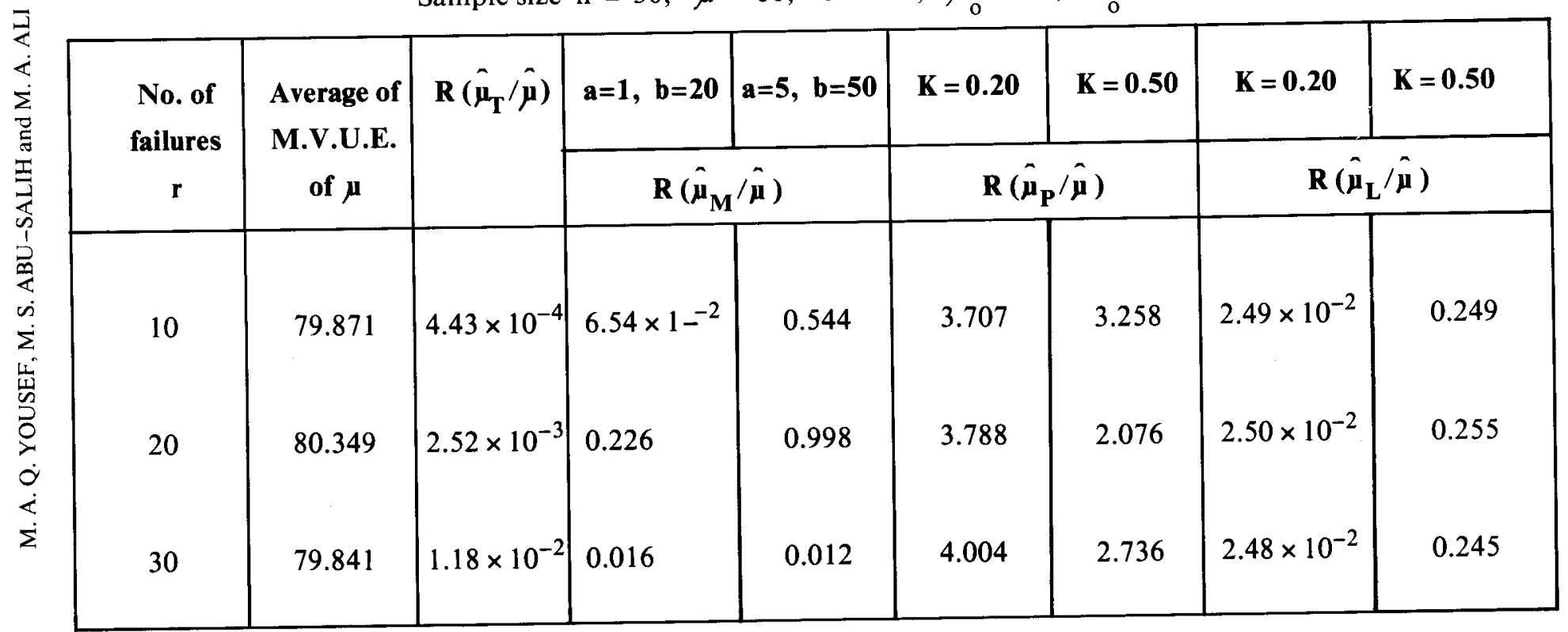


Table 3

Relative Efficiencies of Various Shrunken Estimators of $\theta$

Sample size $n=30, \mu=80, \quad \theta=7.0, \theta_{0}=5.0$

\begin{tabular}{|c|c|c|c|c|c|c|c|c|}
\hline \multirow{2}{*}{$\begin{array}{l}\text { No. of } \\
\text { failures }\end{array}$} & \multirow{2}{*}{$\begin{array}{l}\text { Average of } \\
\text { M.V.U.E. }\end{array}$} & \multirow[t]{2}{*}{$\mathbf{R}\left(\hat{\boldsymbol{\theta}}_{\mathbf{T}} / \hat{\boldsymbol{\theta}}\right)$} & $a=1, b=20$ & $a=5, b=50$ & $K=\mathbf{0 . 2 0}$ & $K=\mathbf{0 . 7 0}$ & $\mathbf{K}=\mathbf{0 . 2 0}$ & $\mathbf{K}=\mathbf{0 . 7 0}$ \\
\hline & & & \multicolumn{2}{|c|}{$\mathbf{R}\left(\hat{\boldsymbol{\theta}}_{\mathbf{M}} / \hat{\boldsymbol{\theta}}\right)$} & \multicolumn{2}{|c|}{$\mathbf{R}\left(\hat{\boldsymbol{\theta}}_{\mathbf{P}} / \hat{\boldsymbol{\theta}}\right)$} & \multicolumn{2}{|c|}{$\mathbf{R}\left(\hat{\boldsymbol{\theta}}_{\mathbf{L}} / \hat{\boldsymbol{\theta}}\right)$} \\
\hline 10 & 7.358 & $9.34 \times 10^{-3}$ & 0.066 & 0.769 & 1.150 & 1.270 & $2.33 \times 10^{-3}$ & 0.095 \\
\hline 20 & 7.338 & $1.48 \times 10^{-2}$ & 0.645 & 1.0 & 3.965 & 1.435 & $2.53 \times 10^{-2}$ & 0.223 \\
\hline 30 & 7.207 & $4.13 \times 10^{-2}$ & 0.876 & 1.0 & 3.978 & 1.753 & $2.50 \times 10^{-2}$ & 0.256 \\
\hline
\end{tabular}




\section{REFERENCES}

Lemmer, H. H. 1981. Note on shrinkage estimators for the Binomial distribution. Commum. Statist. Theory. Meth. A 10 ( 10 ), 1017-1027.

Mehta, J. S.; and Srinivasan, R. 1971. Estimation of the mean by shrinkage to a point Jr. Amer. Statist. Assoc. 66, 86-90.

Pandey, B. N. 1979. On shrinkage estimation of normal population variance. Commun. Statist. Theory. Meth. A 8 ( 4 ), $359-365$.

Pandey, B. N. and Singh, J. 1977. Estimation of variance of normal population prior information. Jr. Indian Statist. Assoc. 15, 141 - 50. 